\title{
Mathematical Wave Fitting Models for the Quantification of the Diurnal Profile and Variability of Pulse Wave Analysis Parameters
}

\author{
Lena G. M. Bauer ${ }^{1 *}$, Bernhard Hametner², Christopher C. Mayer², S. Wassertheurer ${ }^{2}$ \\ ${ }^{1}$ Institute for Analysis and Scientific Computing, TU Wien, Wiedner Hauptstraße 8-10, \\ 1040 Vienna, Austria; ${ }^{*}$ lena.bauer@tuwien.ac.at \\ ${ }^{2}$ Center for Health \& Bioresources, AIT Austrian Institute of Technology, Biomedical Systems, Donau-City-Str. 1, \\ 1220 Vienna, Austria
}

SNE 27(3), 2017, 153-160, DOI: 10.11128/sne.27.tn.10386 Received: February 10, 2017, (Selected ASIM STS 2016 Postconf. Publ.), Accepted: February 25, 2017

SNE - Simulation Notes Europe, ARGESIM Publisher Vienna, ISSN Print 2305-9974, Online 2306-0271, www.sne-journal.org

Abstract. The analysis of 24 hour (24h) ambulatory blood pressure monitoring (ABPM) profiles and their variability has been of interest in literature for considerable time. The development of sophisticated algorithms, which are integrated into mobile sphygmomanometers, allows the performance of $24 \mathrm{~h}$ ABPM including pulse wave analysis (PWA). The recording involves the measurement of standard ABPM parameters as well as the estimation of central aortic pressures and other systemic cardiovascular parameters at regular time intervals throughout the day. The resulting time series often show a diurnal profile. Therefore, the analysis of these profiles and their variability is of interest. In this context, the analysis of diurnal blood pressure (BP) profiles serves as a model. The methods are adapted to be applicable to the time series independent of the parameter. In this article a selection of mathematical models and indices to quantify this profile and the variability of the time series are presented. The considered fitting models are a square wave fit, a fourier fit and a double logistic fit. The modelling process as well as advantages and disadvantages of each method are given. The results show that the algorithms performing the fits are feasible for the $24 \mathrm{~h}$ profiles and provide several indices quantifying certain characteristics of the profiles.

\section{Introduction}

Cardiovascular diseases are one of the leading causes for morbidity and mortality [1]. It is therefore of crucial importance to identify indicators for these diseases at an early stage to find proper treatment and prevent fatal outcome. There are many parameters describing the health condition of the cardiovascular system, the most popular being systolic and diastolic BP. However, hypertension is only able to predict $40 \%$ of coronary heart diseases [2]. Therefore, further indicators have to be found. The availability of oscillometric brachialcuff based blood pressure monitors, which include algorithms estimating central aortic pressures and other systemic cardiovascular parameters, enables the recording of ABPM and PWA parameters at regular time intervals throughout the day. The Mobil-O-Graph (I.E.M., Stolberg, Germany) is an example for such a monitoring device, which includes validated algorithms providing the PWA parameter values [3]. The resulting time series often show a diurnal profile. Therefore, the analysis of these profiles and their variability is of interest. In this context, the analysis of diurnal BP profiles serves as a model. These methods, which have been used in clinical studies for $24 \mathrm{~h}$ BP profiles for considerable time $[4,5,6]$, are adopted for other parameters of the PWA in order to mathematically quantify the variability of a time series regardless of the parameter. The aim of this article is to describe the calculation details of three such methods. All of them are curve fitting models which aim to assess the diurnal profile of the parameter time series. In general, this is achieved by an ansatz function of a specific form, which is fitted to the data set by a least squared error criterion. The advantages and disadvantages of each model are presented as well. The provided variability and profile indices might help to find further indicators for cardiovascular diseases.

\section{Methods}

This section deals with the motivation and calculation details of three fitting models: the square wave fit, the fourier fit and the double logistic fit. 
Least squared error criterion. Let $x_{1}, \ldots, x_{n}$ and $t_{1}, \ldots, t_{n}$ denote the measured values throughout the $24 \mathrm{~h}$ period and the corresponding time points, respectively. In general, the purpose of the curve fitting method is to determine the parameters $\lambda_{1}, \ldots, \lambda_{m}$ of an ansatz function $X_{\lambda_{1}, \ldots, \lambda_{m}}:[0,24) \longrightarrow \mathbb{R} \quad m<n$, which takes certain different forms as the parameters are varied, such that the residual sum of squares

$$
\sum_{i=1}^{n}\left(x_{i}-X_{\lambda_{1}, \ldots, \lambda_{m}}\left(t_{i}\right)\right)^{2}
$$

reaches its minimum.

\subsection{Square Wave Fit}

Motivation. BP tends to vary around a higher level during wakefulness than during night while being asleep in healthy patients $[5,7,8,9]$. The purpose of the square wave model is to capture this characteristic of the diurnal parameter profile. The period times of the higher and lower plateau are determined by the model.

Calculation. The ansatz function for the square wave model [4] is given by

$$
S W(t):= \begin{cases}a, & t \in\left\{t_{i}, t_{i+1}, \ldots, t_{i+k}\right\}, 1 \leq k<n \\ b, & t \in\left\{t_{1}, \ldots, t_{n}\right\} \backslash\left\{t_{i}, \ldots, t_{i+k}\right\},\end{cases}
$$

where $a$ and $b$ are the mean values of the data points $\left\{x_{i}, x_{i+1}, \ldots, x_{i+k}\right\}$ and of $\left\{x_{1}, \ldots, x_{n}\right\} \backslash\left\{x_{i}, \ldots, x_{i+k}\right\}$, respectively. The parameters $i$ and $k$ remain to be determined by the least squared error criterion. For a data set of $n$ measurements there exist $n \cdot(n-1)$ such square waves. In order to obtain the best fit curve with respect to the squared error, all possible square waves as well as the data points themselves are normalized. The data are transformed

$$
x_{1}, \ldots, x_{n} \longmapsto \tilde{x}_{1}, \ldots, \tilde{x}_{n}
$$

with

$$
\tilde{x_{i}}:=\frac{x_{i}-\bar{X}}{c S D} .
$$

The curve is transformed

$$
S W(t) \longmapsto S W_{s t}(t)
$$

with

$$
S W_{s t}(t):= \begin{cases}\frac{a-\overline{S W}}{\sigma_{S W}}, & t \in\left\{t_{i}, t_{i+1} \ldots, t_{i+k}\right\}, 1 \leq k<n \\ \frac{b-S W}{\sigma_{S W}}, & t \in\left\{t_{1}, \ldots, t_{n}\right\} \backslash\left\{t_{i}, \ldots, t_{i+k}\right\}\end{cases}
$$

and with

$$
\begin{gathered}
\overline{S W}=\frac{k \cdot a+(n-k) \cdot b}{n} \\
\sigma_{S W}^{2}=\frac{1}{n-1}\left(k \cdot(a-\overline{S W})^{2}+(n-k) \cdot(b-\overline{S W})^{2}\right) .
\end{gathered}
$$

For each of the standardized square waves the crosscorrelation coefficient is calculated as the average product of corresponding values of the curve and the original data, i.e.

$$
c c_{j}=\frac{1}{n} \sum_{i=1}^{n} S W_{s t}\left(t_{i}\right) \cdot \tilde{x}_{i} .
$$

These $n \cdot(n-1)$ values range from -1.0 to 1.0 , where a low value stands for a poor fit and 1.0 means that the curve is a perfect fit. Therefore, the curve with the highest cross-correlation value is chosen to be the best fit curve (Figure 2).

\subsection{Fourier Fit - Truncated Fourier Analysis}

Motivation. In this approach, a linear combination of cosine waves with different amplitudes and acrophases but known periods are fitted to the data. The motivation for this ansatz is Fouriers perception, that ,... any time series, regardless of its shape or regularity, can be described by a series of sine and cosine waves of various frequencies (Fourier 1822).'[10]

Calculation. The general ansatz in a fourier analysis is given by a fourier series

$$
F(t)=a_{0}+\sum_{k=1}^{\infty}\left(a_{k} \cdot \cos (k t)+b_{k} \cdot \sin (k t)\right)
$$

The model curve which is desired to describe the $24 \mathrm{~h}$ data profile is given by $[10,11]$

$$
\begin{gathered}
f(t):=M+C_{1} \cos \left(\frac{2 \pi t}{24}+\phi_{1}\right)+\cdots \\
\cdots+C_{k} \cos \left(\frac{2 \pi k t}{24}+\phi_{k}\right),
\end{gathered}
$$

where $M$ is called the mesor and $C_{1}, \ldots, C_{k}$ are constants representing the amplitudes of the cosine components. The acrophases (phase shifts, given in 
rad) are indicated by $\phi_{1}, \ldots, \phi_{k}$. As a first observation one sees that a finite number of ansatz functions instead of the infinite series is used (,truncated'). Further, it is sufficient to solely use cosine functions, since a sine function can always be replaced by a cosine function due to the relation $\sin (x)=\cos \left(x-\frac{\pi}{2}\right)$. Furthermore, all constants $C_{1}, \ldots, C_{k}$ can be assumed to be greater or equal to zero, since the sign of the cosine can be changes by a phase shift: $-\cos (x)=\cos (x-\pi)$. The period of the $i-$ th harmonic is equal to $\frac{24}{i}$ hours.

Using the addition theorem $\cos (A+B)=$ $\cos (A) \cos (B)-\sin (A) \sin (B)$ yields

$$
\begin{aligned}
f(t)= & M+C_{1} \cos \left(\frac{2 \pi t}{24}\right) \cos \left(\phi_{1}\right)+\cdots \\
& \cdots-C_{1} \sin \left(\frac{2 \pi t}{24}\right) \sin \left(\phi_{1}\right)+\cdots \\
& +\cdots \quad- \\
& \cdots+C_{k} \cos \left(\frac{2 \pi k t}{24}\right) \cos \left(\phi_{k}\right)+\cdots \\
& \cdots-C_{k} \sin \left(\frac{2 \pi k t}{24}\right) \sin \left(\phi_{k}\right) .
\end{aligned}
$$

The substitutions

$$
\begin{array}{ll}
X_{i}(t)=\cos \left(\frac{2 \pi i t}{24}\right), & a_{i}=C_{i} \cos \left(\phi_{i}\right) \\
Z_{i}(t)=\sin \left(\frac{2 \pi i t}{24}\right), & b_{i}=-C_{i} \sin \left(\phi_{i}\right)
\end{array}
$$

for $i=1, \ldots, k$ then lead to the linear regression model

$$
\begin{aligned}
f(t)=M & +a_{1} X_{1}(t)+b_{1} Z_{1}(t)+ \\
& +\cdots+ \\
& +a_{k} X_{k}(t)+b_{k} Z_{k}(t)
\end{aligned}
$$

The independent variables are here $X_{i}$ and $Z_{i}, i=$ $1, \ldots, k$. The variables $M, a_{i}$ and $b_{i}, i=1, \ldots, k$ have to be determined employing a (weighted) least squared error analysis. The (optional) weights are the lengths of the intervals between two consecutive measurements. The distance is seldom constant [12]. The values for $a_{i}$, $b_{i}$ und $M$ have to be determined in a way, that the residual sum of squares is minimal. In the following, the case is studied, where the sum of squared errors is extended by a weight $w_{i}$ for each data point $x_{i}$. Therefore, the following expression has to be minimized

$$
\begin{aligned}
R S S & =\sum_{i=1}^{n} w_{i}\left(x_{i}-f\left(t_{i}\right)\right)^{2} \\
& =\sum_{i=1}^{n} w_{i}\left(x_{i}-\left(M+\sum_{j=1}^{k}\left(a_{j} X_{j}\left(t_{i}\right)+b_{j} Z_{j}\left(t_{i}\right)\right)\right)\right)^{2} .
\end{aligned}
$$

If calculations should be done without any weighting, all $w_{i}$ can be set to one in the whole scheme. The above error estimate is minimal, if all the derivatives with respect to each parameter are equal to zero. Consider therefore

$\frac{\partial}{\partial M} R S S=\sum_{i=1}^{n} 2 \cdot w_{i}\left(x_{i}-\left(M+\sum_{j=1}^{k}\left(a_{j} X_{j}\left(t_{i}\right)+b_{j} Z_{j}\left(t_{i}\right)\right)\right)\right) \cdot(-1)$.

Setting this expression equal to zero and making the variables of interest, namely $a_{j}, b_{j}$ and $M$, explicit' leads to the first equation

$$
\sum_{i=1}^{n} w_{i} x_{i}=M \cdot \sum_{i=1}^{n} w_{i}+\sum_{j=1}^{k} a_{j}\left(\sum_{i=1}^{n} w_{i} X_{j}\left(t_{i}\right)\right)+\sum_{j=1}^{k} b_{j}\left(\sum_{i=1}^{n} w_{i} Z_{j}\left(t_{i}\right)\right)
$$

The derivatives with respect to the $a_{s}, 1 \leq s \leq k$ and $b_{s}, 1 \leq s \leq k$ yield to further $2 k$ equations. The total of $2 k+1$ equations can be written as a linear equation system in matrix form

$$
S \cdot \vec{l}=\vec{b},
$$

where $S$ is the matrix

$$
\left(\begin{array}{cccccccc}
w_{i} & w_{i} X_{1} & w_{i} X_{2} & \cdots & w_{i} X_{k} & w_{i} Z_{1} & \cdots & w_{i} Z_{k} \\
w_{i} X_{1} & w_{i} X_{1}^{2} & w_{i} X_{2} X_{1} & \cdots & w_{i} X_{k} X_{1} & w_{i} Z_{1} X_{1} & \cdots & w_{i} Z_{k} X_{1} \\
w_{i} X_{2} & w_{i} X_{1} X_{2} & w_{i} X_{2}^{2} & \cdots & w_{i} X_{k} X_{2} & w_{i} Z_{1} X_{2} & \cdots & w_{i} Z_{k} X_{2} \\
\vdots & \vdots & \vdots & \ddots & \vdots & \vdots & \vdots & \vdots \\
w_{i} X_{k} & w_{i} X_{1} X_{k} & w_{i} X_{2} X_{k} & \cdots & w_{i} X_{k}^{2} & w_{i} Z_{1} X_{k} & \cdots & w_{i} Z_{k} X_{k} \\
w_{i} Z_{1} & w_{i} X_{1} Z_{1} & w_{i} X_{2} Z_{1} & \cdots & w_{i} X_{k} Z_{1} & w_{i} Z_{1}^{2} & \cdots & w_{i} Z_{k} Z_{1} \\
\vdots & \vdots & \vdots & \vdots & \vdots & \vdots & \ddots & \vdots \\
w_{i} Z_{k} & w_{i} X_{1} Z_{k} & w_{i} X_{2} Z_{k} & \cdots & w_{i} X_{k} Z_{k} & w_{i} Z_{1} Z_{k} & \cdots & w_{i} Z_{k}^{2}
\end{array}\right),
$$

and in front of each entry of the matrix stands a sum $\sum_{i=1}^{n}$, and each $X$ and each $Z$ has $t_{i}$ as argument. Further,

$$
\vec{b}=\left(\begin{array}{c}
\sum_{i=1}^{n} w_{i} x_{i} \\
\sum_{i=1}^{n} w_{i} x_{i} X_{1}\left(t_{i}\right) \\
\vdots \\
\sum_{i=1}^{n} w_{i} x_{i} X_{k}\left(t_{i}\right) \\
\sum_{i=1}^{n} w_{i} x_{i} Z_{1}\left(t_{i}\right) \\
\vdots \\
\sum_{i=1}^{n} w_{i} x_{i} Z_{k}\left(t_{i}\right)
\end{array}\right) \text { and } \vec{l}=\left(\begin{array}{c}
M \\
a_{1} \\
\vdots \\
a_{k} \\
b_{1} \\
\vdots \\
b_{k}
\end{array}\right) \text {. }
$$


This linear equation system can be written as

$$
\left(X^{T} \cdot W \cdot X\right) \vec{l}=\left(X^{T} \cdot W\right) \vec{x},
$$

where $W=\operatorname{diag}\left(w_{1}, \ldots, w_{n}\right)$ is a diagonal matrix containing the weights, $\vec{x}=\left(x_{1}, \ldots, x_{n}\right)$ is the vector containing the given data and $X$ is the matrix

$X=\left(\begin{array}{ccccccc}1 & X_{1}\left(t_{1}\right) & \cdots & X_{k}\left(t_{1}\right) & Z_{1}\left(t_{1}\right) & \cdots & Z_{k}\left(t_{1}\right) \\ 1 & X_{1}\left(t_{2}\right) & \cdots & X_{k}\left(t_{2}\right) & Z_{1}\left(t_{2}\right) & \cdots & Z_{k}\left(t_{2}\right) \\ \vdots & \vdots & \vdots & \vdots & \vdots & \vdots & \vdots \\ 1 & X_{1}\left(t_{n}\right) & \cdots & X_{k}\left(t_{n}\right) & Z_{1}\left(t_{n}\right) & \cdots & Z_{k}\left(t_{n}\right)\end{array}\right)$.

This representation is simpler to implement. The solution is now given by

$$
\vec{l}=\left(X^{T} \cdot W \cdot X\right)^{-1} \cdot\left(X^{T} \cdot W\right) \vec{x} .
$$

Finally, the desired parameters of the model curve are calculated as

$$
\begin{gathered}
C_{i}=\sqrt{a_{i}^{2}+b_{i}^{2}} \\
\phi_{i}=\left\{\begin{array}{ll}
-\tan ^{-1}\left|\frac{b_{i}}{a_{i}}\right| & b_{i}>0 \wedge a_{i} \geq 0 \\
-\pi+\tan ^{-1}\left|\frac{b_{i}}{a_{i}}\right| & b_{i} \geq 0 \wedge a_{i}<0 \\
-\pi-\tan ^{-1}\left|\frac{b_{i}}{a_{i}}\right| & b_{i}<0 \wedge a_{i} \leq 0 \\
-2 \pi+\tan ^{-1}\left|\frac{b_{i}}{a_{i}}\right| & b_{i} \leq 0 \wedge a_{i}>0
\end{array} .\right.
\end{gathered}
$$

\subsection{Double Logistic Fit}

Motivation. Previously mentioned curve fitting methods partly work under at least one of two non legitimate assumptions.

- The parameter profile is perfectly symmetric. The assumption is that the decline of the parameter shows exactly the same characteristics as its surge (fourier fit, if only one harmonic is used and square wave fit).

- The periods, in which the considered parameter is higher respectively lower have the same length (fourier fit, if only one harmonic is used).

Both assumptions do not reflect reality - at least not for BP, for which the models were developed. The method of the double logistic analysis does not include any of these hypotheses. Head et al. developed this method for heart rate and BP data of rats [13]. In [14] they applied the method to heart data of humans.
Calculation. The model curve which is desired to describe the $24 \mathrm{~h}$ profile is given by $[13,14]$

$$
y(t)=P_{1}+\frac{P_{2}}{1+e^{P_{3}\left(P_{4}-t\right)}}+\frac{P_{2}}{1+e^{P_{5}\left(P_{6}-t\right)}},
$$

where $P_{1}$ to $P_{6}$ have to be determined. Such curves can be shaped as shown in Figure 1, depending on the choice for $P_{1}$ to $P_{6}$. The curve is then fitted to the data with a least squared error criterion.

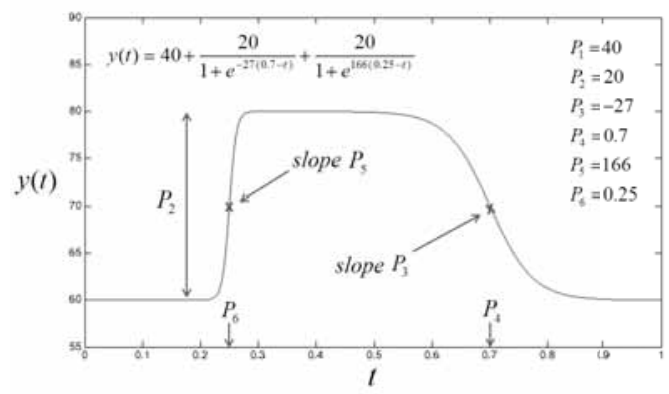

Figure 1: Example of a double logistic curve.

Implementation by Head et al. The model described in $[13,14]$ proceeds more complex as the authors add four terms to the model curve in equation 6 to obtain a quasi periodic function. These additional terms are related to the preceding and the following day. Another term $P_{2} \cdot q$ is added as a compensation parameter. The parameter $q$ is equal to -2 , if the data begin with the transition from high to low. Otherwise $q$ is chosen as 2 . The actual fitting curve therefore takes the form

$$
\begin{aligned}
y(t)=P_{1} & +\frac{P_{2}}{1+e^{P_{3}\left(P_{4}-t\right)}}+\frac{P_{2}}{1+e^{P_{5}\left(P_{6}-t\right)}} \\
& +\frac{P_{2}}{1+e^{P_{3}\left(P_{4}-t-24\right)}}+\frac{P_{2}}{1+e^{P_{5}\left(P_{6}-t-24\right)}} \\
& +\frac{P_{2}}{1+e^{P_{3}\left(P_{4}-t+24\right)}}+\frac{P_{2}}{1+e^{P_{5}\left(P_{6}-t+24\right)}} \\
& +P_{2} \cdot q .
\end{aligned}
$$

This double logistic ansatz function is then fitted by a specially developed computer program written in Labview. It makes use of the Marquardt algorithm, which optimizes the parameters by the least squared error criterion. This requires adequate start values for the variables $P_{1}$ to $P_{6}$. By iteration the parameters are optimized by minimizing the squared error. To obtain first approximations for these values, another fitting method, 
namely the Cosinor model (= fourier fit with one harmonic), is used. For instance, a first approximation for $P_{2}$ is taken as two times the amplitude of the cosinor fit. Furthermore, for the parameters several constraints are made. The limits for $P_{1}$ and $P_{2}$ are determined from the square wave fit. Mean values and standard deviations of the higher level period as well as of the lower level period, according to the square wave, are calculated. Define $y_{\max }$ as the mean of the higher level values plus two times the according standard deviation and $y_{\min }$ as the mean of the lower level values minus two times the according standard deviation. The constraints for $P_{1}$ and $P_{2}$ can then be chosen as

$$
\begin{aligned}
y_{\text {min }} \leq & P_{1}+P_{2}<y_{\text {max }} \\
& P_{2}>0 \\
y_{\text {min }} \leq & P_{1}+2 P_{2}<y_{\text {max }} .
\end{aligned}
$$

Constraints for the curvature parameters were chosen in a way that transition phases lasted for at least 30 minutes. Plateaus should be at least five hours long. Details to the algorithm can be found in $[13,14]$.

Implementation in MATLAB The approach to obtain a double logistic curve fit presented in this section is a simplified version of the one described above. It is done by the use of two different MATLAB built-in functions, namely nlinfit and lsqcurvefit, which fit the function given in formula 6 to the data set by the least squared error criterion. These two functions require start values for the parameters $P_{1}$ to $P_{6}$. They are obtained from the cosinor fit. The slopes at the two inflection points and their according time points are the initial values for $P_{3}$ to $P_{6}$. The level difference $P_{2}$ is chosen as the difference between the high level and the low level period as determined according to the cosinor method. $P_{1}$ is approximated by the difference of the low level mean and the approximation of $P_{2}$.

\section{Results}

Each of the described methods provides several indices quantifying the data profile. They are described in this section and exemplary plots of fitted curves are given.

\subsection{Square Wave Fit}

As can be seen in Figure 2, the square wave provides several indices quantifying the characteristics of the di- urnal profile of the data including the period durations of the higher and the lower plateau as well as the transition time points and the level difference.

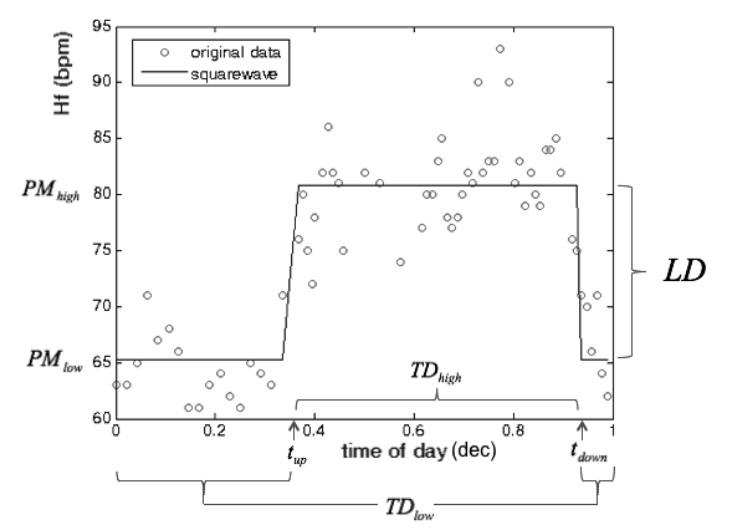

Figure 2: Square wave fitted to a data set of $24 \mathrm{~h}$ heart frequency $(\mathrm{Hf})$ data. The determined parameters $t_{i}=t_{u p}$ and $t_{i+k}=t_{d o w n}$ indicate the time points of the transition from the lower plateau to the higher plateau and vice versa. Further, the mean values in the periods $P M_{\text {high }}$ and $P M_{\text {low }}$, the period durations $T D_{\text {high }}$ and $T D_{\text {low }}$ as well as the level difference $L D$ are shown.

\subsection{Fourier Fit}

Basically the model provides two indices [12], which are graphically shown in Figure 3. As can be seen, the model predicts the occurrence of the maximum value at about 4 p.m., which is very close to the actual maximum. The overall amplitude serves as a measure for the range of the data.

\subsection{Double Logistic Fit}

The approach presented in the paragraph Implementation in MATLAB often yields favourable results for both of the functions nlinfit and lsqcurvefit as can be seen in Figure 4 at peripheral systolic BP as well as Hf data. However, for some data sets the curve is shaped unfamiliarly (Figure 8).

The indices obtained from the model are precisely the parameters $P_{1}$ to $P_{6}$ of the ansatz function. The parameters $P_{1}$ to $P_{6}$ represent the following qualities.

- $P_{1}+P_{2} \ldots$,baseline', , night - time - plateau'; This value is approximately the mean of the data measured during the lower level period. 


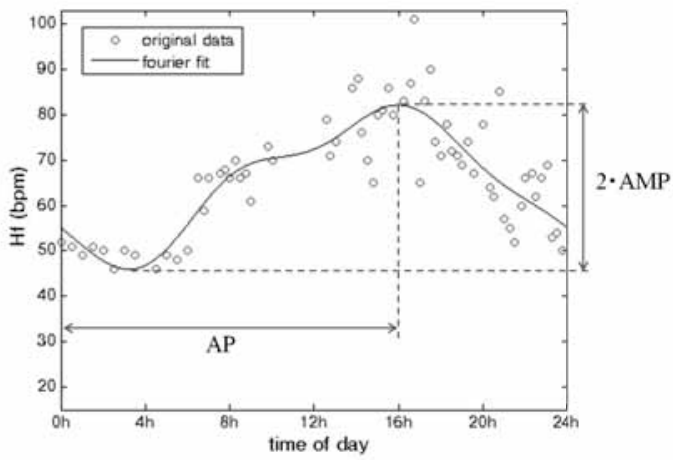

Figure 3: Fourier fit for $\mathrm{Hf}$ data using three harmonics. The parameters of the model are the overall acrophase (AP), which is defined as the time point of the maximal value of the model curve, and the amplitude (AMP), which is defined as half the extent of the range of the data.
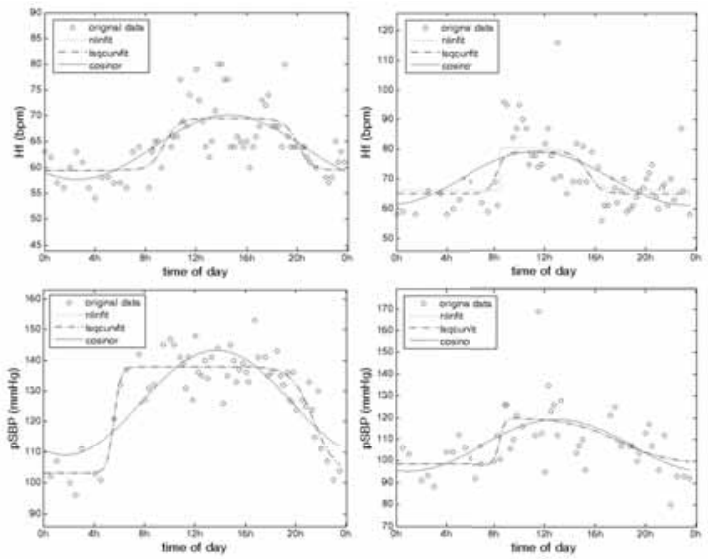

Figure 4: The double logistic functions take reasonable forms for different data sets ( $\mathrm{Hf}$ and pSBP). For the data in the top right corner, nlinfit and lsqcurvefit provide different curves. Nevertheless, both seem comprehensible.

- $P_{2} \ldots$,amplitude'; This represents the range of the data, the difference between the lower level and the higher level period, respectively.

- Accordingly, $P_{1}$ is the lower level value minus the difference of the two plateaus. Therefore, to obtain the approximation of the mean value of the high level period, one has to add the difference of the plateaus $P_{2}$ to the lower level plateau $P_{1}+P_{2}$, which equals $P_{1}+2 P_{2}$.
- $P_{3}$ and $P_{5}$ serve the modelling of the transitions between the plateaus. They indicate the extent of steepness of the change between the levels. While $P_{3}$ is the slope from the higher to the lower plateau, $P_{5}$ gives the slope of the reverse transition.

- The values $P_{4}$ and $P_{6}$ are the time points at which $50 \%$ of the transition is reached. Therefore, they are the middle time points within the transition periods.

Features of the MATLAB algorithm. One of the observations when applying the algorithm described in the section Implementation in MATLAB to different data sets is, that the curve does rise to the higher level but fails to fully return to the lower level plateau as can be seen in Figure 5. To avoid this unfavourable effect, two approaches can be made.
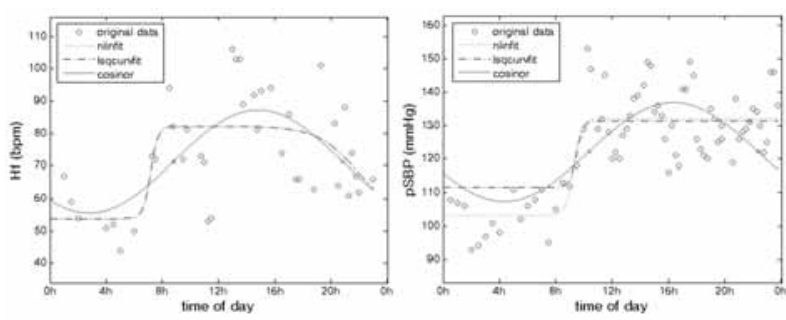

Figure 5: The plots show the unfavourable effect, that the double logistic curve does not return to the lower level plateau.

Since the start of the sleep time lies approximately within the interval $(22 \mathrm{~h}, 2 \mathrm{~h}),(\mathrm{BP})$ values begin to fall rather close to the end of the $24 \mathrm{~h}$ monitoring period. This might hinder the curve to perceive another low level period. To obtain enough lower values, the data set may be extended by a certain number of measurements of the following day. In the absence of these measurements, simply the first couple of hours of the same day with the according measurements are added. Applying the implemented MATLAB function on the data set with an extension of six hours to the same data sets as in Figure 5 leads to the desired return to the lower level plateau. This can be seen in Figure 6 .

The second option is to shift the time point of the beginning of the measurements such that transition periods are most likely not close to the beginning or the end of the observation period. 

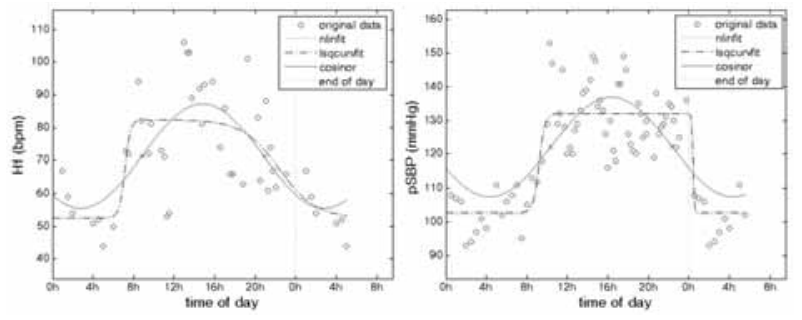

Figure 6: Double logistic curve fit with an extension of six hours to the data set.

Applying the MATLAB algorithm again to the same data sets as in Figure 5 and 6, respectively, with the start time set to 4 p.m. yields to the double logistic curves depicted in Figure 7. However, the shape of the curve is rather sensitive to the starting time, since the fitted functions in 6 and 7 show - at least for the data set on the left - notably different characteristics.
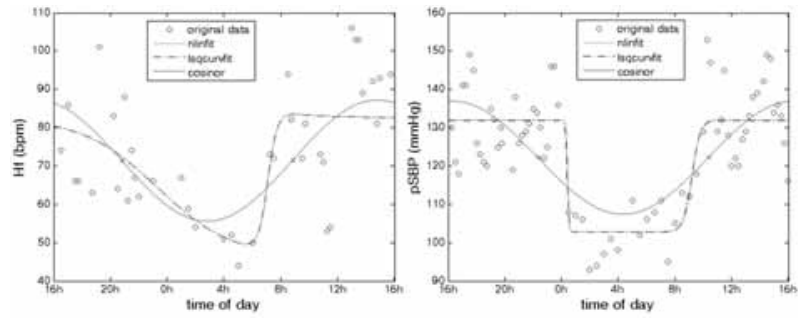

Figure 7: Double logistic curve fit with a shift of the starting time of the observations to 4 p.m.

\section{Discussion}

The advantages and disadvantages of each method are discussed in this section.

\subsection{Square Wave Fit}

This method of capturing the profile of the dataset is a refinement to the so called nocturnal BP fall [12]. There, the averaging of the data points in the alleged higher and lower periods is done over defined day time and night time periods which includes a subjective component. The square wave is advanced in the sense of correctness, since it is a method based on a mathematical model and the periods are implicitly determined [4]. In [15] it is further stated that the square wave approach performs better in fitting BP data as well as the heart rate changes than the Cosinor method.

Although the square wave captures several features of the parameter profile, while the degrees of freedom are limited to the two time points, when the level changes [4], one drawback of this approach is that the ansatz assumes abrupt and symmetrical transition periods. This does not reflect the fact that these transitions vary strongly from subject to subject - at least for BP data - [15].

\subsection{Fourier Fit}

There is no distinct statement which number of harmonics is the best choice. It is conjectured that various numbers of harmonics are possible 'best choices', depending on the (temporal) distance between two measurements [4]. Other authors hold that the model is better the more harmonics are used [11]. However, their recommended number is four harmonics, since the method performed best for different data sets and the influence of added harmonics on the indices of the model were negligible. As well as the square wave also the fourier analysis can be used to segment the $24 \mathrm{~h}$ interval in a lower level and a higher level period. However, Idema et al. [4] claim that the square wave method performs better considering segmentation.

The Fourier method captures the complexity of the signal better than the previously mentioned square wave approach. However, the smoothing effect might lead to an 'over-modelling' of the measurements [15]. Another advantage of this method is that it is applicable to nonequidistant data sets. Additionally, this method does not assume symmetrical period transitions or equal period durations [11].

\subsection{Double Logistic Fit}

The method is said to improve the modelling of the surge of (BP) data in the morning, which is known to be a risk factor for stroke $[13,16]$. The crucial innovation of this method is the possibility to consider the decline of the values and the rise separately [14]. Additionally, the model refrains from symmetry assumptions on the data profile. However, despite the presented favourable results, some further observations have to be mentioned. The implementation of this method by Head et al. $[13,14]$ is rather complex and the design of the curve in general seems to be only applicable to data sets with a specific shape. This can be seen in Figure 8. 
The data sets do not show a typical diurnal BP tenor, which leads to a rather unfamiliar double logistic fit. Head at al. include a lot of restrictions on the parameters of the model. This inclusion might improve the simplified approach presented above. Another observation made when applying the MATLAB algorithms on the data sets is, that the resulting curve is rather sensitive to the initial values. The improvement of the calculation of adequate initial values presents another field of investigation to obtain a solid method.

As the two MATLAB function often provide different results for the data sets, they require further analysis to find distinct quality criteria for the decision in favour of one of them.
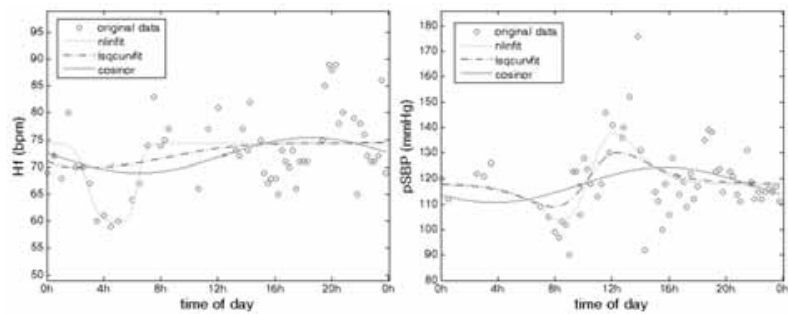

Figure 8: For some data sets the MATLAB algorithms yields to unfamiliar double logistic curves.

\section{Conclusion}

The results show that the algorithms performing the fits are feasible for the $24 \mathrm{~h}$ profiles and provide several indices quantifying certain characteristics of the profiles. Although the double logistic model requires further refinement, the results are encouraging.

\section{References}

[1] WHO-editor. Cardiovascular diseases Fact sheet Updated May 2017. website

http://www.who.int/mediacentre/ factsheets/fs317/en/visited on 09/07/2017.

[2] Gerstenblith G and Margolis S. Coronary heart disease. The John Hopkins White Papers, John Hopkins Medicine, Baltimore, Maryland. 2007

[3] Weber T, Wassertheurer S et al. Validation of a brachial cuff-based method for estimating central systolic blood pressure. Hypertension. 2011; 58(5): 825-832.
[4] Idema R N, Gelsema E S et al. A new model for diurnal blood pressure profiling - Square wave fit compared with conventional methods. Hypertension. 1992; 19(6): 595-605.

[5] Mancia G, Di Rienzo M et al. Ambulatory blood pressure monitoring use in hypertension research and clinical practice. Hypertension. 1993; 21(4): 510-524.

[6] Höcht C. Blood pressure variability: prognostic value and therapeutic implications. ISRN Hypertension. 2013; 2013. doi:

http://dx.doi.org/10.5402/2013/398485

[7] Degaute J-P, Van De Borne P et al. Quantitative analysis of the 24-hour blood pressure and heart rate patterns in young men. Hypertension. 1991; 18(2): 199-210.

[8] Millar-Craig M W, Bishop C N. Circadian variation of blood-pressure. The Lancet. 1978; 311(8068): 795-797.

[9] Sayk F, Becker C et al. To dip or not to dip. Hypertension. 2007; 49(5): 1070-1076.

[10] Refinetti R, Cornélissen G et al. Procedures for numerical analysis of circadian rhythms. Biological Rhythm Research. 2007; 38(4): 275-325.

[11] Thijs L, Staessen J A et al. Analysis of the diurnal blood pressure curve. High Blood Pressure and Cardiovascular Prevention. 1992; 1: 17-28.

[12] Thijs L, Staessen J A et al. Number of measurements required for the analysis of diurnal blood pressure profile. Journal of Human Hypertension. 1994; 8(4): 239-244.

[13] Head G A, Lukoshkova E V et al. Non-symmetrical double-logistic analysis of 24-h blood pressure recordings in normotensive and hypertensive rats. Journal of Hypertension. 2004; 22(11): 2075-2085.

[14] Head G A, Reid C M et al. Nonsymmetrical double logistic analysis of ambulatory blood pressure recordings. Journal of Applied Physiology. 2005; 98(4): 1511-1518.

[15] Parati G. Assessing circadian blood pressure and heart rate changes: advantages and limitations of different methods of mathematical modelling. Journal of Hypertension. 2004; 22(11): 2061-2064.

[16] Kario K, Pickering T G et al. Morning surge in blood pressure as a predictor of silent and clinical cerebrovascular disease in elderly hypertensives. Circulation. 2003; 107(10): 1401-1406. 